\title{
The Idea Arc: Designing a Visual Canvas for Fuzzy Ideas
}

\begin{abstract}
Alejandro Lecuna Aguerrevere
HTW Berlin - University of Ap-

plied Sciences, Berlin. Germany

alejandrolecuna@gmail.com
\end{abstract}

\author{
Katja Thoring \\ Delft University of Technology, \\ Delft, The Netherlands \\ katja@thoring.com
}

\author{
Roland M. Mueller \\ Berlin School of Economics \\ and Law, Berlin. Germany \\ roland.mueller@hwr-berlin.de
}

\begin{abstract}
At the fuzzy front end of innovation emerging ideas are often fuzzy as well. This may lead to premature rejection of those early ideas and hence result in missed business opportunities. This paper introduces the Idea Arc-a canvas-based innovation tool that facilitates team-based discussions, elaboration and refinement of an idea, identification of missing parts, detection and correction of inconsistencies, finding fit between elements, and preparation for prototyping. We developed the canvas based on expert interviews and tested it in two workshops. As a result, this paper contributes to the existing research on the fuzzy front end of innovation and also informs the emerging research on visual innovation tools.
\end{abstract}

\section{Introduction}

It is said that deep inside the Bavarian Forests, in southern Germany, lives a fearsome critter, which the locals refer to as the 'Wolpertinger'. It is a hybrid-like creature with a body comprising of parts from various animals. The head of a jackrabbit, the body from a squirrel, it has antlers similar to those of a deer, eagle wings, the rear legs are from a duck, and the front ones from a chicken. In this odd and absurd looking wild thing, nothing seems to fit. The local folk culture also uses the term 'Wolpertinger' for describing something non-sensical.

Similarly, early born ideas [7] at the fuzzy front end (FFE) of the new product development process (NPD) sometimes look fuzzy or even ugly, just like a Wolpertinger. These fuzzy ideas [21] are characterized by an unfamiliar or illogical appearance, where its defining elements are not connected, missing, or unfitting. In these ideas, the level of uncertainty and ambiguity is high [3], and therefore a slight negativity might be associated with them, which might result in premature rejection of these ideas.

The history of innovations also tells us that many radical ideas often didn't show themselves as beautiful and logic as they looked in the end (if they ever made it to the market). Mostly, they appeared unfinished, absurd, or even ugly in their initial state - and yet they contained seeds for profitable innovations. Think, for example, of the first self-contained filmless camera invented by Kodak in 1975 by Steven Sasson. This 'halfbaked', 'toaster-looking' thing didn't work properly and looked messy (just like a Wolpertinger). The potential of this new technology was not apparent to the managers and decision makers at first sight. The tragic decision from Kodak back then was to postpone this project many times over the years, missing a once-in-a-lifetime opportunity to be the world leader in digital photography. Wrigley and Straker referred to this anecdote as 'Kodak's missed moment' [23:8].

It seems as if few people can sense the potential and beauty behind odd-looking ideas. Most people see only the absurdity and crude reality - the idea looks ugly, it must be a bad idea, so they end up walking away and leaving the Wolpertinger dead in the innovation forest.

Ideas at the FFE are indeed hard to evaluate for many companies because they do not fit the existing 'frame of references' [3]. They lack a clear definition as well as the key elements and crucial information, which is necessary to create a basic common understanding. Consequently, those fuzzy ideas trigger suspicion, negative reactions, and fear from managers, team members, and decision makers, who rather favor ideas that are easy to understand. Hence, they might overlook the chances and opportunities for more radical innovations, which we believe lie inside those fuzzy ideas. But how could companies determine if there is potential in those fuzzy ideas in order not to miss an innovation opportunity? Consequently, with this paper, we address the following research questions:

$R Q$ 1: What elements are important to better understand a fuzzy idea and its context?

$R Q$ 2: How can we develop a visual innovation tool to facilitate the process of transforming fuzzy ideas into mature design concepts?

We introduce a canvas-based innovation tool-the Idea Arc - that facilitates team-based discussions, elaboration of an idea, identification of missing parts, detec- 
tion and correction of inconsistencies, finding fit between elements, and preparation for prototyping. The goal of this canvas is to establish a common language among design teams, to encourage discussions and openness towards uncertainty, to prevent premature rejection of ideas, and to help identifying the hidden potential of fuzzy ideas.

We describe the development process of the canvas based on three expert interviews and evaluate the applicability of the tool in two action research workshops in both, academia and practice contexts. As a result, this paper contributes (1) to the existing literature on innovation at the fuzzy front end of innovation and (2) to the emerging research on visual innovation tools.

\section{Related work}

Our literature review focuses on existing work in two areas: (1) ideas at the fuzzy front end of innovation and (2) visual facilitation and co-creation tools, such as canvases. For both areas we conducted a snowball search within the Scopus database. The insights from the related literature informed our own development process of a canvas for elaborating and refining ideas at the FFE of innovation.

\subsection{Ideas at the fuzzy front end of innovation}

The innovation process is often associated with the design thinking process [e.g. 4]. However, we argue that design thinking constitutes only one possible approach to innovation. Innovative ideas can also emerge through other channels and approaches, for example through ideas individually developed by employees. Hence, we refer to the more general innovation process model suggested by Koen et al. [14] that divides the typical innovation process into three steps: (1) the fuzzy front end (FFE), (2) the new product development (NPD), and (3) the commercialization of the innovation. Other than the work at the later stages of the innovation process, the fuzzy front end is typically unstructured, often involves interdisciplinary teams, and it is characterized by uncertainty. Nevertheless, it is here, where the true potential for leap innovation lies.

Liedtka [15] outlined various cognitive biases that might lead to flaws in cognitive processing and premature rejection of novel ideas. Bessant [3] elaborated on the importance of selecting innovation projects which are 'out of the box', but pointed out that there is simply not enough information early on to allow an unambiguous choice to be made. For Anderson [1] the lack of clarity in an idea or product concept in the FFE causes a major problem, an impediment for the work, delaying it and even preventing it from proceeding to the next phase. Brown [4] suggested that organizations should embrace an attitude of experimentation and prototyping when sensing and evaluating early born ideas. A similar concept as a fuzzy idea is the so-called 'dark horse'. A dark horse is "a young, unknown horse that gains a surprise win at the races" $[8: 4]$. According to this metaphor, dark horse ideas are not easy to recognize, just like fuzzy ideas. Dark horse prototypes [5] are three-dimensional physical prototypes that are built to explore a previously rejected idea. Karlsson and Törlind [12] elaborated on ideas that have been rejected in the early innovation process. They suggested that those rejected ideas could be seen as a potential goldmine of innovations. These specific characteristics of ideas at the fuzzy front end encouraged our aim to develop a visual tool for interdisciplinary teams to systematically facilitate idea-refinement- that is elaborating and fleshing-out fuzzy ideas.

\subsection{Visual facilitation and co-creation tools}

Our decision to develop a canvas-based tool was informed by Sanders [20], who outlined the development of co-creation over the past thirty years. In [19] Sanders suggested to provide "generative tools for co-designing that enable and facilitate collaborative thinking, mapping, dreaming and storytelling". Moreover, she argued that "a toolkit usually contains a background on which to work, together with a large number of simple and ambiguous components that can be arranged and juxtaposed in a variety of ways". In [19] Sanders emphasized the idea of toolkit-supported "strategic visioning workshops" as the next step of co-creation. Visual tools should enable a group of people to work together and share ideas. Furthermore, visual modeling languages, such as Service Blueprints [22], are well-established to facilitate innovation processes.

We build on research by Eppler, Hoffmann and Bresciani [10] who suggested that collaborative teamwork based on templates can have a positive impact on innovation processes. Eppler, Öste and Bresciani [11] suggested that visual tools can effectively act as collaboration catalysts and are highly appreciated by the participants. Also, Avdiji, Elikan, Missonier and Pigneur [2] suggested to create a shared visualization for collaborative team processes.

The concept of canvases is well-established since the Business Model Canvas [16], which addresses the specific problem of developing business model ideas. Besides that, there exist various tools that offer support on different stages of the new product development process. Roberts [18] presented a list of 50 visual canvases for different areas. There exist several ideation canvases, for example the Innovation Canvas [13], or the Big Idea Canvas [24]. However, these do not address the specific circumstances of ideas at the fuzzy front end. Moreover, as already pointed out by Avdiji et al. [2], 
most canvases are developed by practitioners and hence lack scientific rigor. Our search for 'idea canvas' within the Scopus database (limited to title, keywords, and abstract) returned zero results, which supports this assumption and justifies our attempt to systematically develop and evaluate an idea canvas that addresses idea elaboration at the fuzzy front end of innovation.

\section{Theoretical framework}

Our canvas development process is guided by a conceptual framework that outlines the problems and opportunities behind fuzzy 'Wolpertinger' ideas. It is based on the concepts of false positive and false negative decision errors. False positive (type I) errors lead to investing a lot of resources into projects that in the end fail on the market. In contrast, false negative (type II) errors result in missed business opportunities because the potential of a project or idea has not been recognized. Chesbrough, Vanhaverbeke and West [6:8] point out that companies most often focus on minimizing the risk of false positive (type I) errors, whereas little effort is invested in identifying and reducing false negative (type II) errors.

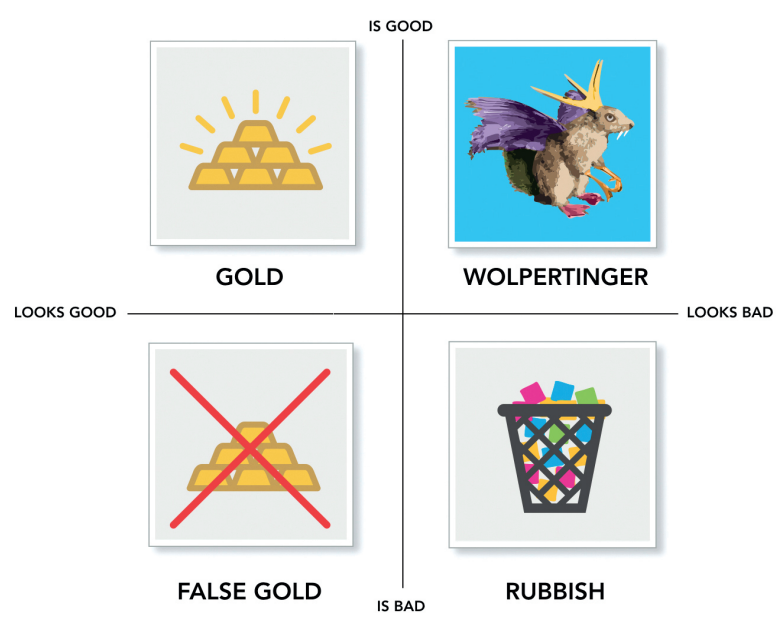

Figure 1: Theoretical framework of fuzzy ideas

Our framework differentiates two dimensions: ideas that look good or bad at first glance, and ideas that actually are good or bad after deep evaluation or in reality. Those ideas that look good and are good (the 'Gold' in our framework) can be recognized by anybody. Often those ideas generate some kind of hype that results in a great deal of companies investing in the same area. We argue that ideas that look good are often foreseeable and hence might only lead to incremental innovations. Those ideas that look good but are bad ('False Gold' in our framework) constitute type I errors. Erroneously investing in those can lead a company to invest lots of time and resources but in the end the ideas will fail on the market. A thoughtful user-centered design thinking process might reduce this risk, because this way user needs could be identified early in the process and be addressed accordingly [4]. Ideas that look bad are of particular interest, because most people will not follow up on them. However, we argue that they deserve deeper investigation. Of course, it is possible that those ideas actually are bad ('Rubbish' in our framework), but there is also the chance they turn out as containing hidden potentials (the 'Wolpertingers' in our framework). The latter constitute type II errors: not identifying their potentials and prematurely rejecting those ideas will inevitably lead to a missed business opportunity.

Figure 1 outlines our suggested framework. If a company was able to detect those hidden potentials of bad-looking, fuzzy ideas, they will probably not have lots of competition at this time, because others will possibly overlook their potential and ignore these ideas. What is needed is a tool that helps companies to distinguish a bad looking idea into 'Wolpertingers' and 'Rubbish', which is the goal of the visual tool presented in this paper.

\section{Methodology}

In order to better understand the underlying problems that companies face when developing ideas at the fuzzy front end of innovation, we conducted three interviews with experts from different institutions. Through these interviews we were able to identify the core elements of fuzzy ideas, typical situations and contexts, as well as problems within the NPD process of the respective companies. We chose this inductive approach because we consider a canvas-based approach to innovation processes a highly practice-related issue, and hence argue that insights from active practitioners would provide us with richer insights than theory alone.

The subsequent development of the suggested canvas was guided by a three-step approach, as suggested by Avdiji et al. [2]: First, we developed an ontology in which the main elements of the idea and their relationships were modeled. Second, we transferred the ontology into a shared visualization to structure the concepts logically into a visual empty problem space. Third, also our canvas suggests a large printout and optional use of post-it notes.

After developing the canvas, we conducted two action research workshops in order to identify potentials for improvement and to evaluate the applicability and usefulness of the canvas within a practitioners' context. 


\section{Expert interviews}

\subsection{Procedure}

We conducted three expert interviews to identify problematic areas within companies' innovation processes, as well as key requirements for a visual tool that could help identifying the potential of early (bad-looking) ideas at the fuzzy front end of innovation. We chose experts who work or consult for international companies that support employees to come up with innovation ideas (employee-generated ideas) at the FFE. The first expert (E1) is a practitioner from a French multinational retail corporation (300,000 employees). His position is that of a Corporate Learning Chief Officer, who is responsible for promoting and teaching innovation processes throughout the organization. Because of the highly dynamic nature of the retail sector, his organization has to constantly come up with innovative ideas and solutions. The second expert (E2) is a practitioner from a European insurance company (25,000 employees) in the position of Head of Development \& Strategy. The insurance business is highly conservative, where risk management prevails over creativity. Therefore, innovations happen rarely and are regarded with caution. The third expert (E3) is a design innovation consultant and facilitator working for several multinational companies (from 50 to 200,000 employees) in various economic sectors. Additionally, E3 works as a coach and workshop facilitator in the academic world. Consequently, E3 has a broader and more comparative perspective on innovation. All experts had an average of more than 10 years of relevant professional experience. Because of the different perspectives those three experts have on innovation and idea development at the FFE, we consider them as corner cases. The interviews were semi-structured and based on the following set of open questions:

- How does your company come up with ideas?

- What are the known problems in that process?

- How do you engage employees into innovation?

- How do you prototype ideas?

- What tools and methods are you using?

- What happens to rejected ideas?

- How do you identify the potential of ideas?

The three interviews resulted in a total of 165 minutes of audio data (an average of 55 minutes per interview). The main insights were transcribed and clustered according to appearing patterns.

\subsection{Interview results}

In the following subsections we present the results from the expert interviews that guided our canvas development process according to four emerging themes.

5.2.1. Idea overload. Companies have to innovate continuously to remain competitive; this is the case for all three experts and the companies they represent or advise. For doing so, they mainly rely on their employees to generate ideas. E1 does this since the foundation of the company. They run innovation education programs, provide online idea-suggestion platforms, and they organize offline idea-sharing events, idea contests, and idea micro-accelerator programs. As a result, E1 employees feel at ease at coming up with ideas regularly. However, this creates an 'idea overload'.

Quote 1: "Some of those ideas are okay, others are just repetitions, others are direct copies from our competitors, others make sense only for the local culture or branch but not for the whole company, others sound too complicated, hard to understand" (E1).

The problem for E1 is not coming up with ideas, but how to proceed and evaluate them and how to distinguish between good and bad ideas. E2 also collects ideas from their employees by encouraging them to propose and promote ideas, but they don't have a satisfying systematic way for doing so.

Quote 2: "the problem with these idea boxes is that you end up with too many 'one-liner ideas' [very short description of an idea], those end up being a stone in the shoe, how do you proceed with them?" (E2).

This tells us that such idea suggestion boxes only work when they are combined with a change in the organization's culture and innovation mindset.

Quote 3: "ideas are nothing in themselves, 'they are just an idea'. Ideas need an 'idea hero', an outspoken person that takes ownership of his, or someone else's idea and pushes it, on his own and with his connection through the whole company, all the way to the decision makers at the top." (E2)

Similarly, most of E3's companies employ space interventions like idea suggestion boxes or online platforms for discovering employee-generated ideas, however, she is not aware of what happens to those ideas or how they are being filtered.

Quote 4: “...the companies I consult for prefer to start by detecting design challenges and running innovation workshops around those...". E3)

But the problem remains that the number of ideas generated in those workshops is quite high; selecting the right ideas is still often the real challenge. 
5.2.2. Idea selection. The following interview quotes provide insights on the idea selection processes in organizations.

Quote 5: "in order to select ideas, I categorize ideas first in themes (buckets) and then select ideas with potential through dot voting." (E3)

However, E3 acknowledges that creating themes and then doing dot-voting is an easy but very inefficient way of selecting ideas. She strongly favors selecting ideas by discussing them in depth and then reaching consensus, but achieving this is very time-consuming, especially when there are a lot of ideas on the table.

Only E1 reported having a systematic idea evaluation system in place.

Quote 6: "When we select ideas, we first look at the added value for the company, second at the added value for the customers, third at the know how in the field of activity, fourth at the maturity of the market, and finally at technology feasibility". (E1)

However, they use these evaluation criteria carefully, only as an orientation tool.

Quote 7: "These evaluation criteria are good for detecting the potential of innovations that relate back to things we are already doing [step innovation]. However, when an idea is radical [leap innovation], these criteria are not so good... therefore we use these only as a rough indication". (E1)

Consequently, besides these criteria they involve regional managers for evaluating ideas who also rely on their gut feeling for sensing ideas which they think have potential.

Quote 8: "Ideas move up in the organization mainly depending on the employees' internal connections and support from their direct managers. At the end the board makes the decision if an idea is going to become a project or not." (E2)

Quote 9: "the decision makers decide which idea to go for in a non-transparent or obscure way". (E3)

This seems to be again a case of gut feeling when selecting ideas. E3 also tells us that managers tend to favor ideas coming from employees that have a command of good presentation skills. Ideas coming from employees and teams that don't have this kind of soft skill tend to be overlooked. Selecting ideas seems to represent a problem for most companies; they use a mix of evaluation systems, dot voting, and gut feeling, partly based on 'soft' aspects like personal connections inside the organization or presentation skills of the team.

5.2.3. Idea parts. As explained before, employee generated ideas tend to be conceived from their own point of view and are usually incomplete and missing defining elements
Quote 10: "these one-liner ideas are problematic for us... they are often just repetitions of existing ideas... furthermore they are always incomplete, lacking information, like for who specifically is it for? which is the problem? what is the value of the idea all about? why is it important for our company overall strategy?" (E2)

For E1 the discussions, prototyping, and testing activities are fundamental to bring clarity to ideas.

Quote 11: "An idea should change our customers in a positive way, create value for them, and that becomes apparent only when you prototype ideas. In the past, we had 'non-obvious' ideas, where nobody saw the potential. But after the team discussed the idea and conducted prototyping activities against users, the idea's potential was suddenly obvious to everyone in the organization". (E1)

E2 runs a system of innovation workshops, where employee-generated ideas are fleshed out and accelerated.

Quote 12 "During these cycles of workshops, employees gather around one idea and push it forward by discovering or inventing missing parts-intended user, need, insight, problem, value...". (E2)

5.2.4. Gap between ideas and prototyping. E1 denotes ideas as being 'weak ideas', regardless if they scored high or low in the pre-evaluation. This means they were unsure if there was potential in them or not.

Quote 13: "in order to understand an idea deeply, it needs to be prototyped and tested against customers... on the streets and as early as possible. Firsthand user information is highly important for us and for our decision makers. The prototyping activity for us is a way of researching the users and their life... but getting employees to go out is not easy, they discuss and think too much what they want to prototype". (E1)

Quote 14: "A prototype is any kind of physical thing that attempts to explain an idea and that gives you feedback, for example, a 2-dimensional sketch on paper". (E3)

For E1 and E3 early customer feedback is highly valuable, because it can help teams to gather firsthand information, understand users, finetune, and change a fuzzy idea until it makes sense.

Quote 15: "Sometime the idea changes so much based on user's feedback that it is no longer the same idea we started with". (E1)

All experts acknowledged the importance of prototyping, but they all tell us that teams consistently hesitate to prototype and they often consume a lot of time deciding what to prototype and how to prototype it. 


\section{The Idea Arc canvas}

\subsection{Ontology}

The main insights from the interviews were transcribed and clustered according to emerging patterns, which then led to 14 concepts that appeared to be relevant for the innovation process in the three organizations. Based on this, we developed an ontology that summarizes the 14 concepts as well as relationships among them (Figure 2). The 14 concepts are described briefly in the next section. Table 1 summarizes the 14 concepts along with the interview quotes that informed them.

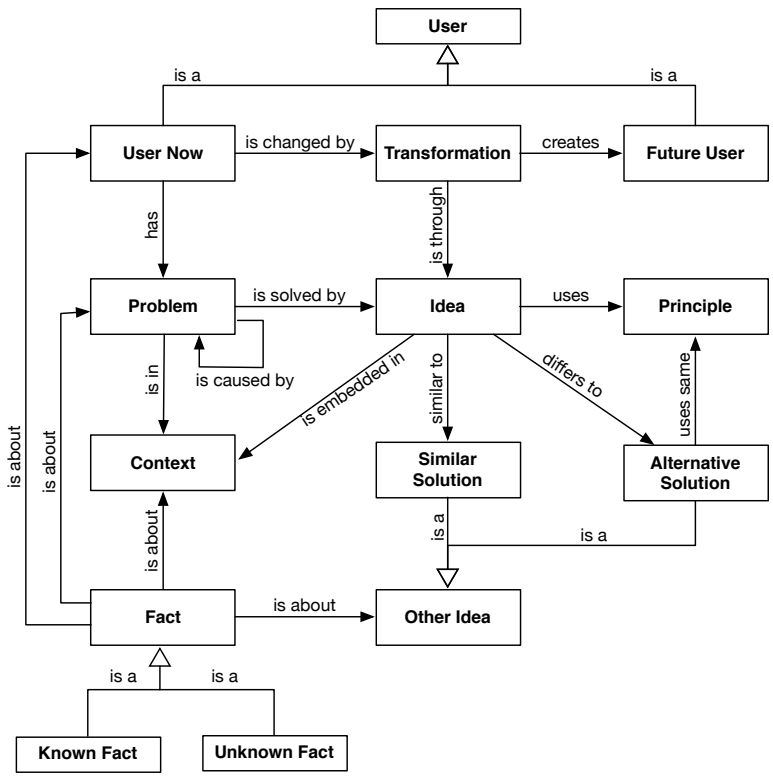

Figure 2. Idea-refinement ontology

1. Idea. This is a short and pragmatic description of what the idea is and how it works.

2. Principle. The principle describes what the idea enables someone to do or achieve (value).

3. Problem. An idea deals with a series of primary and secondary problems that occur within a specific context.

4. User. Description of the personal characteristics and the world-view of an ideal user.

5. User now. Description of the user's needs (wishes, desires) before his or her needs have been fulfilled.

6. Future user. Description of the user's life after his or her needs have been fulfilled.

7. Transformation. This is the desired user change.

8. Facts. Pieces of information.

9. Known facts. Pieces of information that are known about the idea.

10. Unknown facts. Pieces of information that are not known about the idea and must be researched.
11. Other ideas. Ideas that are different from the idea that is being discussed.

12. Similar ideas. Ideas that already exist in the market and are similar to the idea that is being discussed.

13. Alternative solutions. New ideas that address the problem and user in a different way.

14. Context. The territory where the idea operates. It is described through the following questions: (a) Where, when and how was this idea conceived? (b) What is this idea connected with? (c) What aspect is truly unique or special about this idea? (d) Who or what is this idea disrupting? (e) What are the possible complications that this idea will generate when implemented?

Table 1. Relations of concepts and quotes

\begin{tabular}{lll} 
& Concept & Quote Number \\
\hline \hline 1 & Idea & $2,3,10,11,13$ \\
\hline 2 & Principle & $6,10,11,12$ \\
\hline 3 & Problem & 10,12 \\
\hline 4 & User & $6,10,11,12,13$ \\
\hline 5 & User now & $6,11,13$ \\
\hline 6 & Future user & 10,12 \\
\hline 7 & Transformation & 11,15 \\
\hline 8 & Facts & $10,14,15$ \\
\hline 9 & Known facts & $13,14,15$ \\
\hline 10 & Unknown facts & $2,6,10$ \\
\hline 11 & Other ideas & $1,10,15$ \\
\hline 12 & Similar ideas & 1,10 \\
\hline 13 & Alternative solutions & 15 \\
\hline 14 & Context & $8,10,12,13,15$ \\
\hline
\end{tabular}

\subsection{Idea Arc canvas}

The overall goal of the developed visual tool is to provide a platform for discussing, elaborating, and refining fuzzy ideas. In order to facilitate a team discussion and idea refinement process, we suggest a canvasbased tool. The concept of canvases is well-established since the Business Model Canvas [16]. We build on research that suggests that collaborative teamwork based on templates can have a positive impact on innovation processes [10]. We created the canvas by translating the previously developed ontology into visual elements and boxes. We decided to show all the elements of an idea orchestrated around a visual metaphor depicting a user before and after crossing a bridge. Eppler [9] discussed the advantages of visual metaphors in templates for the communication of knowledge. The bridge metaphor helps us to depict the underlying design hypothesis, showing a user before and after the design intervention. The suggested canvas is meant to transform fuzzy ideas into mature design ideas by facilitating team-based dis- 
cussions, refinement of an idea, identification of missing parts, detection and correction of inconsistencies, finding fit between elements, and preparation for prototyping. To use the suggested canvas, the participants need to bring in one fuzzy idea and follow the instructions, as outlined below. These instructions are partly printed on the canvas and additionally explained by the workshop conductors. Figure 3 shows the Idea Arc canvas and illustrates the different idea elements, which were derived from the ontology.

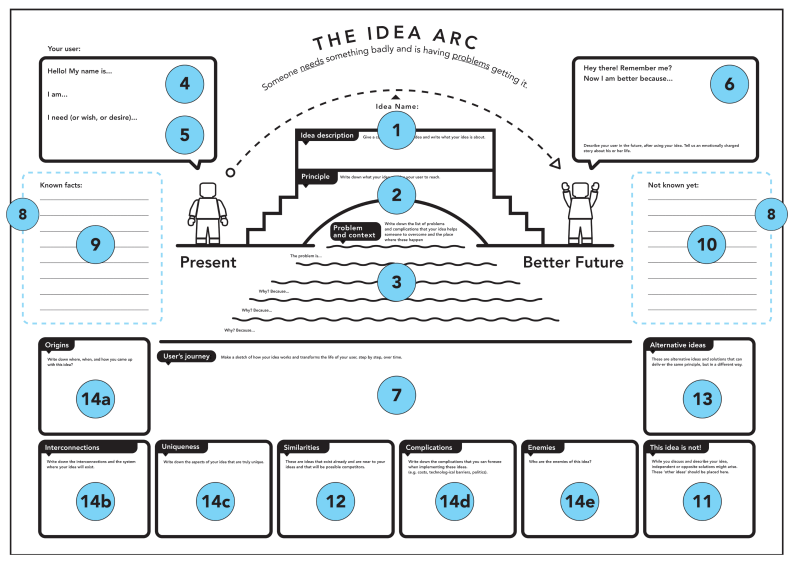

Figure 3: The Idea Arc Canvas

1. Idea Name and description. Give a catchy name to your idea and write what your idea is about.

2. Principle. Write down what your idea enables your user to reach.

3. Problem. Write down the list of problems and complications that your idea helps someone to overcome and the place where these happen.

4. User. Describe your user, in the present, before using your idea. Give him or her a name. Tell us what he or she does.

5. Present User. Write his or her unfulfilled needs, wishes, or desires.

6. Better Future User. Describe your user in the future, after using your idea. Tell us an emotionally charged story about his or her life.

7. User's Journey. Make a sketch of how your idea works and transforms the life of your user, step by step, over time.

8. Facts. These two lists on the sides will serve you to take notes about the pieces of information that you know (9) or still need to find out (10).

9. Known facts. Use this note to keep track of the pieces of valuable information that you know about the idea.

10. Not known yet. Use this note to keep track of the assumptions and pieces of information that you still need to find out.
11. This idea is not. While you discuss and describe your idea, independent or opposite solutions might arise. These 'other ideas' should be placed here.

12. Similarities. These are ideas that exist already and are near to your ideas and that will be possible competitors.

13. Alternative ideas. These are alternative ideas and solutions that can deliver the same principle, but in a different way.

14a. Origins. Write down where, when, and how you came up with this idea?

14b. Interconnections. Write down the interconnections and the system where your idea will exist.

14c. Uniqueness. Write down the aspects of your idea that are truly unique.

14d. Complications. Write down the complications that you can foresee when implementing these ideas. (e.g. costs, technological barriers, politics).

14e. Enemies. Write down the possible enemies of your idea (e.g. competitors).

\section{Evaluation workshops}

To test and evaluate the comprehensibility and practicality of the canvas, we conducted two workshops. The first workshop took place in an academic context during an international design thinking conference. The purpose of this workshop was to evaluate the usability of the canvas - that is, if the participants understood how to operate it. The second workshop took place in an industry context, specifically in an international retail group. The purpose of the industry workshop was to observe the actual usefulness and impact of the canvas on real ideas. Both workshops and the respective observations are described in the following sub-sections.

\subsection{Academic workshop}

7.1.1. Academic workshop procedure: The 75-minute workshop was conducted on-site at an international design thinking conference. 40 participants attended the workshop. They came from different countries and had different professional backgrounds. Among the participants were academics from the design field as well as innovation designers, design managers, design thinking facilitators, and design thinking enthusiasts. At the beginning of the workshop, a 15-minute input was given, in which the participants were introduced to the topic of fuzzy ideas using the Wolpertinger metaphor, as well as to the main canvas components and working mechanisms, using AirbBnB as an exemplary case. Each of the six teams of 6-7 people had the task to elaborate and refine a fuzzy idea using the canvas. We created a fictitious Gym company called 'Gymmie' and presented a series of invented 'employee-generated' fuzzy (one- 
liner) ideas. These ideas were: The 'Gym Van': a gym that comes to your doorsteps. 'Motel Gym': a private gym room with all the necessary equipment and a personal trainer. 'Gym Night club': a gym where you can do sports and meet people. 'Gym Box': rent the gym equipment and have it delivered to your home in a box. 'Face gym': exercise facial muscles to better express feelings and emotions. And 'Gym buddy': an app for helping you pair with someone while being at the Gym. Each team received a short one-liner description of the idea and a printout of the Idea Arc canvas to work on their ideas for 30 minutes. Afterwards the teams presented the ideas and answered questions from the audience.

7.1.2. Academic workshop observations: The workshop was conducted and evaluated by three researchers who took photographs and notes of their main observations and of the arising questions and comments by the audience. They were observing how people used, discussed, and interacted with the canvas and each other during the team exercise. All team members were engaged, intervening, and showing motivation and humor while discussing the ideas. All teams managed to complete the upper part of the canvas, however only one team completed the context questions, which indicated that the allocated 30 minutes were not sufficient to complete the entire canvas, at least not for a group that is not familiar with the tool. The 'user's journey' area was rarely completed. Two teams struggled to agree and find a starting-point; these ones required the presence of a coach in order to give them clear instructions. Particularly the order in which to proceed the different boxes was not fully understood. Four participants struggled to understand the intention of the 'known facts' and 'not known facts' areas. Teams were given freedom to work with or without Post-it notes. It was observed that teams using Post-its covered the questions and prompts printed on the canvas, which made it difficult re-visit areas and to present the canvas to the audience. During the workshop a lot of alternative ideas appeared and were noted in the canvas. These were ideas that shared the underlaying principle, but the design artefact was different. This could suggest that while trying to "vertically brainstorm' an idea, alternative solutions will arise in the process. However, there was not enough space in the box provided on the canvas to archive those emerging alternative ideas.

\subsection{Industry workshop}

7.2.1. Industry workshop procedure: We conducted an industry workshop in order to observe and evaluate the canvas' usefulness and impact on real fuzzy ideas. The chosen company is a French international retail group. The company preselected 6 employee-generated ideas which they considered to be in a 'weak' state. Specifically, they were unsure if there was potential in those ideas or not. Concerns were raised because the framing of the ideas was not convincing and they lacked a clear user, problem, context, and value. The intention of the workshop was to create a common (team) understanding of the ideas and to build prototypes in order to test and validate the ideas with customers.

32 participants attended the workshop from the company side: six idea-conceivers (employees who came up with an idea individually), 24 co-creators (employees who attended the workshop to elaborate and refine ideas as team members), and two workshop coordinators/innovation managers. The participants came from different countries in Europe. From the research side, there were six coaches who facilitated and observed the process and took notes, one lead coach who introduced the theoretical foundations of the Idea Arc, and one lead researcher who observed and recorded the videos.

The 3-day workshop started with the idea-conceivers pitching their ideas to the other participants. Multidisciplinary teams were formed around each of the ideas. The canvas' main components and its usage were presented to the audience. The teams used the canvas to discuss and flesh out the ideas for three hours. After that time, teams re-pitched the ideas to the audience. During the following three days, the teams proceeded to prototype and test the ideas with users and iterate them three times. The information on the canvas was actively updated in each of the iterations based on teams' discussions and feedback from users.

7.2.2. Industry workshop observations: The workshop was evaluated in two ways. (1) The idea pitches were video recorded, before and after using the canvas on the first day, for later analysis. (2) The coaches and the researcher took notes of their observations.

The type of ideas that the participants worked with were ideas that the company considered to be in a fuzzy state, where the value for the user was not fully apparent. During and after running the fuzzy ideas through the canvas and workshop, it was observed that the ideas were easier to understand. This became obvious after reviewing the video presentations before and after using the Idea Arc. It was also observed that when preparing for prototyping, the teams used the Idea Arc to figure out what exactly they wanted to prototype and what they wanted to prove and test with the prototype. During the discussion of ideas, some team members were inspired by the discussions and developed alternative ideas.

Three of the idea-conceivers were observed to be fixed in the way they personally understood their ideas at the beginning of the workshop. But when using the 
Idea Arc, two of them became more open to reconsider and agree to change their original idea. Only one idea conceiver remained strongly attached to his idea and the team could only manage to slightly change it, but no major improvement was observed. Nevertheless, the majority of idea conceivers was able to significantly iterate and improve their ideas through the canvas, up to a point where the whole team started to believe in that idea. During the workshop one project coordinator from the company, who is also innovation manager, pointed out:

Quote 16: "This time, teams are working faster than in prior design innovation workshops, more effectively, they are talking a lot but also doing a lot".

\subsection{Workshop results}

The results from both workshops showed that the proposed canvas supports our initial goals as stated in the research questions. The academic workshop provided useful insights on how to improve the layout and design of the tool, which will be addressed in the next iteration of the canvas.

In the industry workshop, feedback from participants and managers revealed the potential impact on the companies' innovation strategies. For example, three months after the industry workshop, the company informed us that two of the original six ideas (previously considered as 'weak') had been considered for realization by the board of regional directors and that one of them is currently being realized. Therefore, we argue that the canvas can support the NPD process by bringing clarity and acceleration to ideas at the FFE (inferred from Quote 16).

Finally, the observations from the industry workshop indicate that most of the idea-conceivers were willing to change their ideas and include radical improvements when using the canvas. Hence, we argue that the proposed canvas might help in the process of overcoming idea fixation [17]. However, further research is needed to validate this assumption.

\section{Conclusions}

\subsection{Summary}

This paper introduces a visual canvas to elaborate on fuzzy ideas. We developed the tool based on three expert interviews and tested it in two workshops in different contexts. With this study, we addressed two research questions. The first question related to the elements that are important to understand a fuzzy idea and its context. We were able to answer this question through our expert interviews, which provided insights on the innovation practices and related problems in different organizations. The factors that constitute a fuzzy idea could be identified and were addressed in an abstracted ontology. Specifically, we discovered that there are several recurrent elements and questions that people address when discussing, presenting, and describing ideas. We suggest that that if one or more of these elements is not present, the more uncertainty (fuzziness) there will be about an idea. Answering and finding fit between these elements early on in the process can have a positive impact in the way people perceive, judge, and prototype ideas.

The second research question addressed the need for a tool to facilitate idea refinement processes in order to identify hidden potentials in fuzzy ideas. This research question was addressed by a design science approach in which a visual canvas was developed and subsequently tested and evaluated in two workshops, which revealed insights on (a) the usability and understandability of the canvas and (b) the usefulness of the approach for real organizations.

The suggested canvas contributes to the field of innovation methods by facilitating innovation activities in companies, specifically helping them to bring clarity to employee-generated ideas. Furthermore, the canvas could help companies to detect strategic interest areas hiding behind fuzzy ideas. Ideas, in this case, could be seen as carriers not only of potential innovations but also of crucial information, insights, and seeds that could be used to feed strategic decisions and inspire future innovation activities. The suggested canvas could also be implemented by companies to spread a common (design) language for discussing ideas. By simplifying the idea refinement process the canvas encourages employees to suggest their ideas more often. Moreover, employees will learn the language of design and improve their communication skills and hence present their ideas better. However, further research is necessary to validate these assumptions. Furthermore, the research presented in this paper contributes to the emerging research on visual innovation tools by suggesting a rigorous design and development process of a visual canvas. The described process can also guide other researchers when designing or evaluating such tools.

\subsection{Limitations and future work}

The suggested canvas was built based on the insights extracted from only three interviews. However, the three experts all have extensive experience with innovation processes within their respective multinational organizations. They represent three different perspectives from different sectors that can be regarded as corner cases: one conservative global insurance company, one progressive retail chain, and one innovation consultant from industry and academia. Hence, we argue that we 
were able to cover the most important concepts through theses corner cases. However, further research is needed to validate the transferability of the canvas to other contexts. The research resulted in a positive feedback from both workshop's participants but at the same time revealed many insights on how to further improve the tool. Specifically, the canvas could be made more self-explanatory, for example by providing printed instructions on how to use it and proposing a numbered path to be followed when working on an idea. Furthermore, the number of boxes needs to be reduced. The area for sketching the user's journey needs to be revised or left out. A more central role should be given to an 'Alternative Solutions' area, as well as the possibility to modularize the idea into smaller parts. Some boxes might need different labels to improve comprehensibility. Future work will focus on designing the next iteration of the canvas. Moreover, we will look for theoretical evidences in related literature to align our ontology with theory. Further testing of the canvas will include additional workshops within a broader context of general innovation processes, for example after the ideation phase within a design thinking project. Finally, surveys and follow-up interviews shall be conducted in order to further evaluate if the Idea Arc actually has a significant impact on fuzzy ideas and the overall innovation process at the fuzzy front end.

\section{References}

[1] Andersson, S., The fuzzy front end of product innovation processes: the influence of uncertainty, equivocality, and dissonance in social processes of evolving product concepts, PhD Thesis, Luleå University of Technology, Luleå, Sweden, 2010.

[2] Avdiji, H., D. Elikan, S. Missonier, and Y. Pigneur, "Designing Tools for Collectively Solving Ill-Structured Problems", Proceedings of the 51st Hawaii International Conference on System Sciences, (2018).

[3] Bessant, J., B.V. Stamm, K.M. Moeslein, and A.-K. Neyer, "Backing outsiders: selection strategies for discontinuous innovation", R\&D Management 40(4), 2010, pp. 345356.

[4] Brown, T., Change by design: how design thinking transforms organizations and inspires innovation, Harper Business, New York, 2009.

[5] Carleton, T., and W. Cockayne, "The Power of Prototypes in Foresight Engineering", Proceedings of ICED 09, (2009).

[6] Chesbrough, H.W., W. Vanhaverbeke, and J. West, eds., Open innovation: researching a new paradigm, Oxford University Press, Oxford, 2006.

[7] Clapham, M.M., "The Development of Innovative Ideas Through Creativity Training”, In The International Handbook on Innovation. Elsevier, Amsterdam, 2003, 366-376.
[8] Dark Horse Innovation, Digital Innovation Playbook, Murmann Publishers GmbH, Hamburg, 2017.

[9] Eppler, M.J., "The image of insight: The use of visual metaphors in the communication of knowledge", Proceedings of I-KNOW, (2003), 2-4.

[10] Eppler, M.J., F. Hoffmann, and S. Bresciani, "New business models through collaborative idea generation", International Journal of Innovation Management 15(06), 2011, pp. 1323-1341.

[11] Eppler, M.J., H.F. Öste, and S. Bresciani, “An Experimental Evaluation on the Impact of Visual Facilitation Modes on Idea Generation in Teams", 2013 17th International Conference on Information Visualisation, (2013), 339344.

[12] Karlsson, A., and P. Törlind, "What happens to rejected ideas?: exploring the life of ideas following the completion of projects", International Conference on Engineering Design, Design Research Society (2013), 229-238.

[13] Kline, W.A., C.A. Hixson, T.W. Mason, et al., "The Innovation Canvas in entrepreneurship education: Integrating themes of design, value, and market success", The Journal of Engineering Entrepreneurship 5(1), 2014, pp. 80-99.

[14] Koen, P.A., G.M. Ajamian, S. Boyce, et al., Fuzzy front end: effective methods, tools, and techniques, Wiley, New York, NY, 2002.

[15] Liedtka, J., "Perspective: Linking Design Thinking with Innovation Outcomes through Cognitive Bias Reduction", Journal of Product Innovation Management 32(6), 2015, pp. 925-938.

[16] Osterwalder, A., and Y. Pigneur, Business Model Generation: A Handbook for Visionaries, Game Changers, and Challengers, Wiley, Hoboken, NJ, 2010.

[17] Purcell, A.T., and J.S. Gero, "Design and other types of fixation", Design studies 17(4), 1996, pp. 363-383.

[18] Roberts, A., "Canvas collection I - A list of visual templates", Andi Roberts, 2016. http://masterfacilitator.com/canvas-collection/

[19] Sanders, E.B.-N., "Generative Tools for Co-designing", In S.A.R. Scrivener, L.J. Ball and A. Woodcock, eds., Collaborative Design. Springer, London, 2000, 3-12.

[20] Sanders, E.B.-N., "Information, inspiration and co-creation", Proc. 6th International Conference of the European Academy of Design, (2005).

[21] Schreiner, L.C., P.T. Nascimento, and M. Thomaz, "Fuzzy Front End integration: Describing roles and defining boundaries on multi companies", International Conference on Management of Engineering and Technology (PICMET), IEEE (2016), 996-1010.

[22] Shostack, G.L., "Designing Services That Deliver", Harvard Business Review, 1984, pp. 133-139.

[23] Wrigley, C., and K. Straker, Affected: Emotionally Engaging Customers in The Digital Age, Wiley, Melbourne, Australia, 2018.

[24] "Big Idea Canvas", https://www.bigideacanvas.com (last accessed 2018-09-05) 